Die FMH fordert in ihrem Positionspapier, dass jegliche Werbung, jegliche Promotion und jegliches Sponsoring für Tabakprodukte verboten wird. So soll insbesondere Kindern und Jugendlichen den Weg in eine rauchfreie Zukunft erleichtert werden. Wie nötig ein umfassendes Verbot ist, wird im nachfolgenden Artikel dargelegt: Kinder und Jugendliche sind das bevorzugte Zielpublikum der ausgeklügelten Werbeanstrengungen der Tabakindustrie. Wie lange wollen wir dem noch zuschauen?

Dr. med. Christine Romann, Mitglied des Zentralvorstandes der FMH, Departementsverantwortliche Gesundheitsförderung und Prävention

\title{
Tabakwerbung: an jeder Ecke lockt versteckt die Zigarette
}

Iris Leu

Wissenschaftliche Mitarbeiterin Abteilung Gesundheitsförderung und Prävention

Das Rahmenübereinkommen der WHO zur Eindämmung des Tabakgebrauchs sieht verschiedene Massnahmen vor, unter anderem ein umfassendes Tabakwerbeverbot. Die Schweiz hat dieses Abkommen zwar unterzeichnet, aber nicht umgesetzt. Die folgende Zusammenfassung einer Beobachtungsstudie gibt Einblick in die Strategien des Tabakmarketings und zeigt, wie omnipräsent Tabakprodukte in der französischen Schweiz sind im Vergleich zu anderen Ländern.

Die Tabakindustrie kennt verschiedene Marketingstrategien: Tabakwerbung, -verkaufsförderung und -sponsoring. Das Rahmenübereinkommen der WHO zur Eindämmung des Tabakgebrauchs (FCTC) bildet das erste weltweite Gesundheitsabkommen als Antwort auf die Globalisierung der Tabakepidemie. Es enthält konsequente Massnahmen wie ein Verbot für Tabakwerbung, -verkaufsförderung und -sponsoring. Damit dies wirksam ist, muss das Verbot alle Formen des Marketings einschliessen. In der Definition des FCTC ist dies der Fall:

- Tabakwerbung und Tabakverkaufsförderung: alle Formen von Kommunikation, Empfehlungen oder Verkaufshandlungen mit dem Ziel, der Auswirkung oder der potentiellen Auswirkung, ein Tabakprodukt oder den Tabakkonsum direkt oder indirekt zu fördern.

- Tabaksponsoring: Beiträge jeglicher Art für ein Ereignis, eine Aktivität oder eine Person mit dem Ziel, der Auswirkung oder der potentiellen Auswirkung, ein Tabakprodukt oder den Tabakkonsum direkt oder indirekt zu fördern.

Die Schweiz hat das FCTC-Übereinkommen zwar 2004 unterzeichnet, aber als eines der wenigen europäischen Länder nicht ratifiziert. Die aktuelle Gesetz- gebung für Tabakwerbung in der Schweiz ist lückenhaft und von Kanton zu Kanton verschieden geregelt. Gewisse Kantone handhaben die Gesetzgebung zu Tabakwerbung, -verkaufsförderung und -sponsoring strenger als andere [1]. Dies gibt der Tabakindustrie grossen Handlungsspielraum. Aus diesem Grund ist

Tabakmarketing ist in der Schweiz im Vergleich zu den meisten europäischen Ländern überall präsent.

Tabakmarketing in der Schweiz im Vergleich zu den meisten europäischen Ländern überall präsent. Nur gerade Radio- und Fernsehwerbung ist hierzulande verboten.

Der Konsum von Tabak wird durch Tabakwerbung erwiesenermassen erhöht. Bei jährlich 9000 Todesfällen infolge von Tabakkonsum in der Schweiz ist Rauchen die wichtigste vermeidbare Todesursache. Dies ist der

\section{Die Beobachtungsstudie}

Die vollständige Beobachtungsstudie finden Sie unter: www.beobachtung-marketing-tabak.ch/ $\rightarrow$ Präsentation $\rightarrow$ Informationsmaterial $\rightarrow$ Präsentationsbroschüre mit den Resultaten des Projekts 
Grund, weshalb die nachfolgend präsentierte Beobachtungsstudie zu Tabakmarketingstrategien lanciert wurde.

Durch subtile Marketingstrategien der Tabakindustrie fällt es kaum jemandem auf, dass Tabakwerbung omnipräsent ist. Man findet sie in Bars, Discos, Kinos, in der Presse, an Musikfestivals, Verkaufsstellen oder bei privaten Anlässen, die von der Tabakindustrie organisiert und gesponsert sind. Für das Zielpublikum, Raucherinnen und Raucher sowie Jugendliche, ist die Verkaufsförderung klar erkennbar, für den Rest der Bevölkerung bleibt sie hingegen eher diskret.

Die empirischen Beobachtungen zeigen, dass die Tabakindustrie ihre Strategien besonders auf Jugendliche ausrichtet. Die Orte, an denen Werbung geschaltet wird, die Sprache und die Gestaltung sowie Bilder zielen darauf ab, Jugendliche anzusprechen. Die Zigarette wird mit Bezugssystemen (Party, Risikofreude, Flirt, Urlaub usw.) in Verbindung gebracht, die bei jungen Menschen positive Assoziationen auslösen. Jugendliche sind empfänglicher für Werbebotschaften, dies zeigen 18 Langzeitstudien [2] mit über 27000 jugendlichen Nichtrauchern zwischen 8 und 18 Jahren, die belegen, dass die Konfrontation mit Tabakwerbung die Wahr-
Das Bundesamt für Gesundheit (BAG) schätzt den Gesamtbestand an Zigarettenautomaten in der Schweiz auf 18000 Stück; in mehreren europäischen Ländern (Frankreich, England, Irland und Griechenland) wurden sie verboten.

scheinlichkeit, mit dem Rauchen zu beginnen, erhöht. Im Rahmen der Studie wurden die Elemente mit Direktwerbung im Alltag eines Jugendlichen erfasst. Während eines Wochenendtages mit üblichen Aktivitäten und einem Abendausgang wird ein Jugendlicher in der französischen Schweiz mit durchschnittlich 68 tabakfreundlichen Reizen konfrontiert.

In der Schweiz haben die Tabakmarken eigene Websites, auf denen sie für ihre Produkte werben und Informationen zu ihren Aktivitäten aufschalten. Dabei ist zu betonen, dass von den in der Studie offiziell erfassten 22 Tabakmarken-Websites 12 für Minderjährige zugänglich waren.

Die Tabakwerbung ist auch in den sozialen Netzwerken stark präsent. Die Tabakindustrie verwendet diese Kommunikationsplattformen, um Werbung und Verkaufsförderung für ihre Produkte zu betreiben. So werden auch von den Nutzern Tabak-Werbebotschaften aufgegriffen und weitergeleitet. Die sogenannten

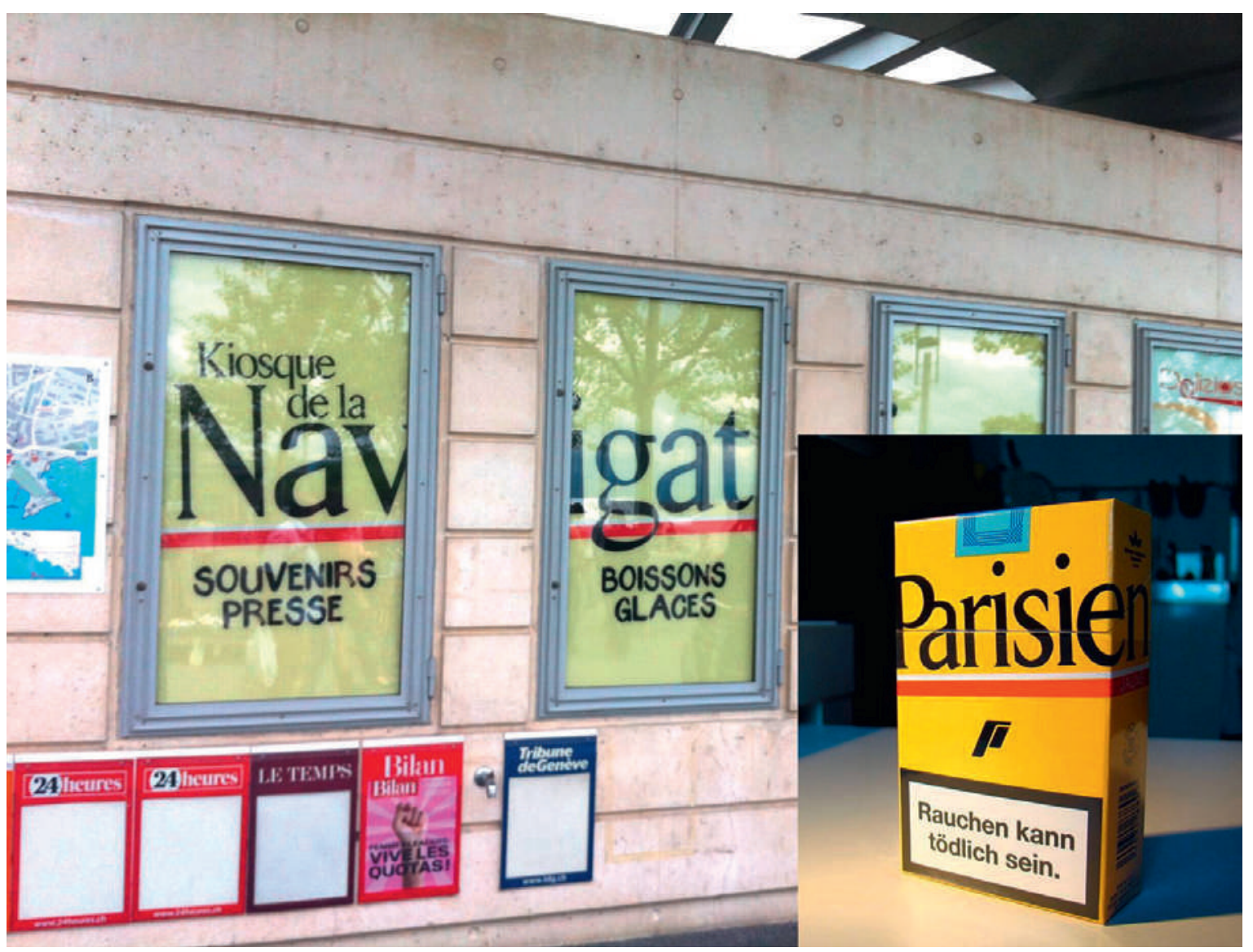

Abbildung 1: Hinweis auf die Marke Parisienne - ein Beispiel für versteckte Werbung. 
Smoking Reviews auf Youtube sind dabei besonders auffällig. In diesen Videos testen die oft sehr jungen Konsumenten die Produkte und inszenieren sich als Raucherinnen, was die Coolness der Zigarette als solche unterstreicht.

Die Marketingformen sind sehr innovativ. Die Tabakwerbung beschränkt sich nicht auf die klassischen Formen (Plakatkampagnen oder Pressewerbung), sondern setzt vielmehr auf Erlebnismarketing, personalisiertes Marketing, Mitmach-Marketing und versteckte Werbung.

\section{Mit einem umfassenden Werbeverbot wird der Tabakkonsum in Ländern mit hohem Einkommen um durchschnittlich 7 Prozent gesenkt.}

Das Ziel von Erlebnismarketing ist es, den Personen ein einmaliges Erlebnis, eine besondere Konsumerfahrung zu bieten, indem die Sinne geweckt werden (festliche Speisen und Alkohol, positive Emotionen in Verbindung mit der Art der Veranstaltung, usw.) oder die Erfahrungen mit starkem emotionalem Charakter (Risiken eingehen, Normen sprengen, etc.) inszeniert werden. Dies ermuntert zum Tabakkonsum. Personalisiertes Marketing zielt darauf ab, den Kunden persönlich zu erreichen, indem es der Konsumentin das Gefühl verleiht, in ihrer Beziehung zur Marke einmalig und privilegiert zu sein. Beim Mitmach-Marketing gilt der Ansatz, die Konsumenten bei der Produktentwicklung oder der Verbreitung von Nachrichten unter anderen Konsumierenden eine Rolle spielen zu lassen. Mit dem Ziel, dass die betroffene Konsumentin in ihrem Umfeld mehr Gehör erhält, als wenn die Marke selber kommuniziert. Versteckte Werbung verwendet gestalterische Elemente (Schrift, Farbgebung usw.), ohne dass der Markenname selbst erwähnt wird. Daraus entsteht ein Vertrautheitseffekt und der Wunsch nach dem Produkt wird erhöht (Abb. 1).

Korrespondenz:

FMH

Elfenstrasse 18

CH-3000 Bern 15

Tel. 0313591111

Fax 0313591112

praevention[at]fmh.ch
E-Zigaretten erregen immer mehr Aufmerksamkeit. Bislang sind diese aber nicht mit Tabakwaren gleichzustellen, weshalb die Werbung dafür nicht geregelt ist. Die Beobachtungen zeigten, dass die Mehrheit der Käufer von E-Zigaretten erwachsene Rauchende sind, die ihren Konsum der klassischen Zigarette verringern oder einstellen möchten.

Aufgrund der Revision des Lebensmittelgesetzes (LMG) wurde ein eigenständiges Tabakproduktegesetz erarbeitet. Im Sommer 2014 ging das Tabakproduktegesetz in die Vernehmlassung.

Der Vorentwurf des Bundesrates enthält verschiedene Verbesserungen gegenüber dem heutigen Gesetz. So will er neu die Tabakwerbung in den Printmedien, auf Aussenplakaten und im Kino untersagen. Aus Sicht der Tabakprävention sollte das Werbeverbot jedoch in allen Bereichen gelten, damit die beabsichtigte Wirkung erzielt werden kann.

In vielen Ländern ist Tabakwerbung, -verkaufsförderung und -sponsoring bereits in jeglicher Form untersagt. Tabakwerbeverbote zeigen eine deutliche Wirkung. Mit einem umfassenden Werbeverbot wird der Tabakkonsum in Ländern mit hohem Einkommen um durchschnittlich 7 Prozent gesenkt. Nur strikte Verbote zeigen aber Wirkung, Teilverbote sind insofern unnütz, da die Werbemittel, die in einem bestimmten Bereich nicht mehr eingesetzt werden können, in einem anderen Bereich genutzt werden, wo Werbung noch erlaubt ist.

\section{Referenzen}

1 Vgl. Tabelle der aktuellen Gesetzgebung zu Tabakwerbung, -verkaufsförderung und -sponsoring in der Schweiz, aus der Beobachtungsstudie der Marketingstrategien in der französischen Schweiz. 2013-2014. S. 9.

2 Lovato $\mathrm{C}$ et al. Impact of tobacco advertising and promotion on increasing adolescent smoking behaviours. Cochrane Database of Systematic Reviews. 2011;5(10).

Das Projekt zur Beobachtung der Marketingstrategien für Tabakprodukte wurde vom Tabakpräventionsfonds finanziert und von CIPRET-Vaud in Zusammenarbeit mit Sucht Schweiz und der Fachstelle Tabakprävention Freiburg-CIPRET umgesetzt. Man kann davon ausgehen, dass die Beobachtungen in der Westschweiz mit der Deutschschweiz vergleichbar sind 